Itinéraires Itinéraires

Littérature, textes, cultures

2019-2 et 3 | 2019

Corps masculins et nation : textes, images, représentations

\title{
La scène, l'écran : questionnements identitaires et tensions du désir dans Tom à la ferme de Michel Marc Bouchard et de Xavier Dolan
}

Stage and Screen: Identitarian Questioning and Tensions of Desire in Michel Marc Bouchard's and in Xavier Dolan's Tom à la ferme

\section{Stefano Genetti}

\section{OpenEdition}

Journals

Édition électronique

URL : http://journals.openedition.org/itineraires/7091

DOI : 10.4000/itineraires.7091

ISSN : 2427-920X

Éditeur

Pléiade

Référence électronique

Stefano Genetti, « La scène, l'écran : questionnements identitaires et tensions du désir dans Tom à la ferme de Michel Marc Bouchard et de Xavier Dolan », Itinéraires [En ligne], 2019-2 et 3 | 2019, mis en ligne le 04 décembre 2019, consulté le 10 décembre 2020. URL : http://journals.openedition.org/ itineraires/7091 ; DOI : https://doi.org/10.4000/itineraires.7091

Ce document a été généré automatiquement le 10 décembre 2020.

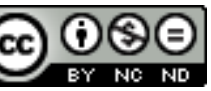

Itinéraires est mis à disposition selon les termes de la licence Creative Commons Attribution - Pas d'Utilisation Commerciale - Pas de Modification 4.0 International 


\title{
La scène, l'écran : questionnements identitaires et tensions du désir dans Tom à la ferme de Michel Marc Bouchard et de Xavier Dolan
}

\author{
Stage and Screen : Identitarian Questioning and Tensions of Desire in Michel \\ Marc Bouchard's and in Xavier Dolan's Tom à la ferme
}

Stefano Genetti

« Ring the bells that still can ring Forget your perfect offering There is a crack in everything That's how the light gets in » Leonard Cohen, Anthem

\section{Rencontre et transfert}

Janvier 2011, au Théâtre d'Aujourd'hui de Montréal, l'une des salles où, dès la fin des années 1960, se fait l'histoire du répertoire dramatique québécois: le jeune cinéaste Xavier Dolan assiste à la représentation de Tom à la ferme, la nouvelle pièce de Michel Marc Bouchard'. Également commissaire d'expositions thématiques où il expérimente une approche théâtrale de la muséologie, Bouchard est depuis plus de vingt ans un dramaturge prolifique et reconnu. Ancré dans l'histoire, dans la société et dans la langue du Québec, son théâtre en transcende les particularités au sein d'une mythologie personnelle de la blessure fondatrice. Traduits et joués dans le monde entier, ses textes ont été souvent adaptés pour le grand ou le petit écran et même mis en musique ${ }^{2}$. Talent flamboyant de la cinématographie québécoise, Xavier Dolan n'est pas encore le réalisateur acclamé et controversé qu'il est devenu avec Mommy, puis avec Juste la fin du monde, d'après la pièce de Jean-Luc Lagarce et, plus récemment, avec The Death and Life of John F. Donovan et Matthias \& Maxime. Son cinéma allie à la 
théâtralité une technique qui, selon le critique Joachim Lepastier, tient du " pompier pyromane ", tant elle est tiraillée entre l'esthétisme, voire la virtuosité, et l'émoi, «entre le visuel et le viscéral » (Lepastier 2014: 8, 9). Du drame de l'homosexualité déniée de Bouchard, où l'humour alterne avec le pathos, Dolan tire l'année suivante un thriller psychologique marquant une rupture de ton par rapport à ses trois premiers films. Le réalisateur écrit le scénario et les dialogues en collaboration avec le dramaturge et il interprète le rôle du protagoniste aux côtés de Pierre-Yves Cardinal, alors que les mêmes comédiennes incarnent les deux personnages féminins au théâtre et lors du tournage ${ }^{3}$. Dans ce cas, la transposition de la scène qui sonde les dégoûts et les dégâts de l'homophobie à l'écran, où sont projetées les apories du désir, comporte de nombreux glissements, dont cette comparaison vise à mettre au jour quelques implications en se concentrant sur la manière dont la pièce d'abord, puis le film, problématisent les questions de l'identité. Celles-ci sont envisagées à l'intersection de considérations d'ordre socio-culturel et linguistique, mais surtout en termes de genre, d'orientation sexuelle et de pluralité du désir, suivant une trajectoire qui, de la résistance contre l'assujettissement et du processus de subjectivation gay, conduit à la mise en cause de la notion d'identité homosexuelle au sein de la pensée queer ${ }^{4}$.

2 Mais la fable avant toute chose. Aller-retour depuis Montréal : un jeune citadin - Tom, « [a]djoint à l'artistique dans une boîte de pub » (Bouchard 2012 : 14) - se rend en pleine campagne pour participer aux obsèques de son amant Guillaume, décédé dans un accident de la route. À la ferme laitière, il rencontre pour la première fois la mère veuve, Agathe, à qui Guillaume n'avait jamais parlé de lui, et le frère aîné de son compagnon, Francis, dont Tom ignorait l'existence. Très attaché à son petit frère, auquel il ressemble beaucoup et avec qui il fréquentait les cours de danses de salon du village, Francis a toujours tout fait pour que leur mère ne sache rien de l'homosexualité de Guillaume. Les cahiers de celui-ci, contenant des poèmes et des dessins « avec des hommes » (19), lui en avaient fourni la preuve. Neuf ans auparavant, lorsque Paul, le petit ami adolescent de son frère, avait voulu lui parler de leur amour - «C'est délicat » (35) -, Francis lui avait ouvert la bouche de ses deux mains et lui avait déchiré le visage en public. À la suite de cet épisode, réglé en privé, moyennant une somme d'argent, Guillaume part à Montréal en s'éloignant d'une famille désormais tenue à l'écart par la communauté rurale environnante. Afin de maintenir leur secret, Francis arrache à son frère une photo où il pose avec une collègue, Sara, qu'ils rebaptisent Ellen - « [b]londe, vingt ans, fume trop, parle pas français » (16) - et qu'ils font passer pour la fiancée de Guillaume. Face à cette situation, Tom, incapable de prendre la parole aux funérailles de son amant, s'enlise dans une spirale de mensonges et développe avec le frère de son amant une relation où convergent la domination et l'assujettissement, la répugnance et l'empathie, voire l'identification et la dépendance réciproques. Poussé par Francis, Tom fait venir Sara à la ferme pour qu'elle incarne aux yeux d'Agathe le rôle d'Ellen, la fiancée imaginaire et pseudo-anglophone du défunt. Excédée, Sara finit par révéler à Tom que Guillaume était un menteur compulsif qui couchait à droite et à gauche, aussi bien avec des garçons que des filles, y compris avec elle. Sous la menace, elle continue pourtant de jouer la comédie jusqu'au moment où Agathe, en sortant de sa propre chambre la boîte où se trouvent les cahiers que son fils lui avait laissés et qu'elle n'a jamais lus, entrevoit enfin la vérité. C'est à partir de ce moment que les intrigues de l'hypotexte dramatique et de l'hypertexte filmique divergent radicalement ${ }^{5}$. 


\section{Une méta-tragicomédie de l'homosexualité dé-mentie}

3 Évocation dé-folklorisée et symbolique du terroir et de son bestiaire, depuis l'orignal esquivé par Tom sur la route jusqu'aux coyotes torturant un lièvre en passant par la fosse aux vaches mortes au-dessus de laquelle Francis suspend Tom, la tête à l'envers, comme il l'avait fait avec Guillaume; assimilation de l'imaginaire religieux dont l'empreinte est tournée en dérision : à plusieurs reprises la mère cite la passion du Christ en identifiant Tom à son fils ressurgi, et, à chaque fois, Francis, puis Tom aussi, de répondre "Amen, m'man », "Amen, Agathe » (Bouchard 2012:21, 42) ${ }^{6}$; contraste des registres et cohabitation de choix dramaturgiques renvoyant d'une part à la tradition québécoise du théâtre domestique et du drame familial, de l'autre au tragique visionnaire. À ces aspects s'ajoute la recherche d'une musicalité verbale toute en reprises et en contrepoints, accentuée par les listes des synonymes qui caractérisent l'idiolecte de Tom. Celui-ci étant la conscience centrale du drame, son discours intérieur, aussi contradictoire que riche en effets comiques, est verbalisé tout au long de la pièce sans pour autant s'adresser directement au public tel un aparté, ce qui donne à voir et à entendre la multiplication des instances énonciatives - l'auteur, le personnage, l'acteur - de la parole théâtrale. Ce sont là autant d'éléments qui font de Tom à la ferme une pièce emblématique de la production de Bouchard, en qui on voit souvent un héritier de Michel Tremblay. Si Bouchard poursuit une critique acérée de la société québécoise en sondant la fatalité des familles - mères justicières, pères absents ou brutaux, inceste, adoption -, son écriture restitue les cadences et les inflexions de l'oralité sans avoir recours au joual, le sociolecte issu de la culture populaire montréalienne. Comme chez la plupart des dramaturges de la génération postréférendaire, les questions identitaires se posent chez lui en termes moins politiques qu'intimes, et si la figure de l'homosexuel, souvent superposée à celle de l'artiste, s'avère dominante dans son œuvre, Bouchard refuse l'étiquette d'écrivain gay ou engagé. Ce qui n'empêche pas Tom à la ferme d'être une pièce sur la persistance de la violence homophobe, dont on risque de sous-estimer l'actualité à une époque où la répression hétéronormative a cédé la place à la gestion sociale des minorités sexuelles (Corriveau et Daoust 2011). Originaire de la région agricole et reculée du Lac-Saint-Jean - la région de Maria Chapdelaine de Louis Hémon-, l'auteur joue, certes, sur l'écart socio-culturel qui sépare le fermier macho et le gay urbain, en reproduisant les clichés du redneck agressif et de la «tantouze» (Bouchard 2012: 31) «[t]rop Dolce, trop Gabbana » (18) qu'il dénonce : "Qu'ils se débauchent en ville autant qu'ils veulent mais ici, pour le petit peu qu'il nous reste, on va garder ça propre» (57). Toutefois, puisque les gens du village condamnent le geste de Francis en l'isolant, on échappe à toute réduction simpliste du phénomène homophobe au décalage entre ville et province (Julien et Lévy 2007). Significativement élaborée, dès 2009, à partir d'un projet inabouti sur le deuil volé aux homosexuels pendant les années sida ${ }^{7}$, cette œuvre interroge les enjeux complexes de l'interdit hétérosexiste et du désir inavouable en déployant l'éventail des répercussions de l'injure et de la honte tour à tour infligées et assumées «tu peux me dire à quoi il sert, ton sperme? " (Bouchard $2012: 36$ ), demande Francis à Tom - et surtout en explorant le motif, éminemment métathéâtral, du mensonge intériorisé. Dans le « Mot de l'auteur » qui précède le texte, on lit : « Avant d'apprendre à aimer, les homosexuels apprennent à mentir. Nous sommes des mythomanes courageux $»(9)^{8}$. 
4 Tom, Agathe, Francis : chacun son deuil, à part soi. Tous, ils héritent du mensonge que Guillaume a érigé en mode de vie et qui déclenche une dynamique réflexive typique de la dramaturgie de Bouchard dès la pièce qui, en 1987, l'a rendu célèbre : Les Feluettes ou La Répétition - au sens théâtral du terme: en costume et en travesti - d'un drame romantique au sein duquel on met en scène Le Martyre de saint Sébastien de D'Annunzio'. Tout au long de Tom à la ferme, les ressemblances, les faux-semblants et les substitutions alimentent un jeu de rôle auquel contribuent jusqu'aux accessoires et aux odeurs. Francis dicte les règles de ce jeu de rôle, puis les subit, au fur et à mesure que les rapports de pouvoir entre lui et Tom se renversent. De même que celui-ci se change sans cesse, qu'il met les habits de travail de Guillaume pour aller à l'étable, puis revêt le top en velours de soie rouge destiné à une fille du cours de danse, Francis finit par mettre le parfum de Tom, c'est-à-dire le parfum que portait Guillaume : «Tu sens ton frère " (Bouchard 2012: 43). Les dimensions éthique et esthétique du métathéâtre se rejoignent et culminent dans les histoires de confiance, discrétion et respect qu'Agathe elle-même avoue s'être racontées pour ne pas lire les cahiers où son fils a inscrit la seule vérité qu'il a apprise en assistant, inerte, au martyre de Paul, c'est-à-dire à la défiguration, par son propre frère, de son amour : «Je pense qu'il faut jamais dire la vérité. Jamais » (57). C'est un Tom à la fois subjugué et complice - avec son « sourire de salon agricole » il appelle Agathe "m'man » (49) - que Sara essaie en vain de réveiller au réel. À propos du veau dont la naissance l'émeut jusqu'à l'extase - jusqu'aux larmes, dans le film -, Tom lui dit: «Ici, c'est du vrai» (49); et la jeune femme de rétorquer : «Tu m'as fait venir ici pour être la fausse blonde d'un mort puis tu me parles de ce qui est vrai ?» (50). Lorsque l'auteur choisit pour sa pièce le titre Tom à la ferme - un titre bon enfant et bucolique qui se veut un leurre, à l'image d'une action «où les équivalents se déclinent en mensonges" (9) - il écarte entre autres La fabrication des synonymes $^{10}$ : «À l'agence», déclare le protagoniste, "on m'appelle monsieur Synonymes. Je cherche les équivalents. La chose qui est comme la chose mais qui est pas vraiment la chose » (20), celle-ci restant occultée, indicible.

5 Car Tom à la ferme est un drame de la parole tour à tour empêchée et extorquée, étouffée et détournée, différée et équivoque. Un drame dont Guillaume est le co-auteur avec Francis, ce dernier étant aussi le metteur en scène : Francis qui ne connait pas les mots pour demander à son frère, puis à Tom, son remplaçant, de rester ; Francis qui ne parle pas aussi bien que Guillaume, "une brute qui pouvait dire des poèmes » (Bouchard 2012 : 32) ; Francis qui à plusieurs reprises étrangle Tom : «Tu me dis quand arrêter! C'est toi qui décides. [...] T'es fort, mon homme. [...] Respire!» (38); Francis qui du même geste - ouvrir de ses deux mains la bouche jusqu'à déchirer la face impose soit la parole, soit le silence : «Si c'est pas moi qui faisais taire Paul, y en a un autre, un jour, qui aurait fait taire ton petit garçon!» (57), se justifie-t-il devant sa mère. Alors que Tom - le personnage à la parole double, intérieure et extérieure - est l'acteur: «Je t'ai trouvé vraiment bon tout à l'heure», le complimente Francis. Mais Tom est aussi l'interprète au sens de traducteur. Si son parfum est "[j]uste un réflexe " (19), sa voix est juste un écho résonnant dans la brèche entre les monologues et les dialogues. "Aujourd'hui, c'est une partie de moi qui meurt et puis j'arrive pas à pleurer. Je connais pas tous les synonymes de la tristesse. Vide, solitude, colère, colère, encore colère! » (16). Attendu par Agathe, commandé par Francis, le petit discours que Tom n'arrive pas à tenir à l'église, la vérité qu'il ne peut pas dire " à la face du monde " (18), Monsieur Synonymes le prononce en l'attribuant à Ellen, Elle-n, une Elle quelconque, dont l'identité est aussi factice que la langue dans laquelle elle est censée 
s'exprimer, c'est-à-dire l'anglais: "Today, a part of me is dying» (22). C'est la conversation téléphonique avec la fausse fiancée du mort que Francis oblige Tom à inventer et à rapporter à Agathe, et c'est par le même biais que Tom évoque le souvenir de la «[b]aise torride» (23) avec son amant: «Toute qu'une cochonne!» (33), cette Ellen, s'esclaffe Agathe. Tandis que Tom singe l'«anglais approximatif » (10) de la francophone Sara, «la verbomotrice alcoolique » (25), celle-ci se met tant bien que mal dans la peau de l'anglophone présumée Ellen et fait semblant de ne pas mieux parler le français qu'Agathe ne comprend l'anglais. S'ensuivent les quiproquos, les fausses traductions et les malentendus révélateurs. En même temps que Michel Marc Bouchard en exploite le burlesque, la surconscience linguistique infranationale, due à la confrontation permanente du québécois avec le français européen d'une part, et avec l'anglo-américain de l'autre, se met au service d'un dispositif métathéâtral où parodie d'un bilinguisme dysfonctionnant et simulation identitaire s'emboîtent l'une dans l'autre ${ }^{11}$. Seule l'écriture échappe au mensonge, mais la vérité inter-dite dont elle est porteuse reste ensevelie dans une boîte à chaussures contenant les cahiers d'un jeune homme mort.

6 Miniature du placard dans lequel la culpabilisation intériorisée cache le désir défendu, cette boîte - véritable moteur de l'action dramatique - se prête dans la pièce à plusieurs associations symboliques: en premier lieu, avec la fosse aux carcasses de vaches «personne va vouloir aller fouiller là-dedans » (Bouchard 2012 : 17); en deuxième lieu, avec le cercueil où gît le cadavre de Guillaume - «La boîte au bois lustré comme un parquet de HLM. [...] dedans la boîte, toi " (18) -, cette métonymie du secret et du cercueil se poursuivant dans la scène du cimetière, sur la tombe du garçon, au cours de la confrontation entre Francis - «Fallait pas lire ça!» (56) - et Agathe, mater dolorosa moins lacrimosa que véhémente; en troisième lieu avec le coffre de la voiture où, pendant la «baise torride » de Francis avec Sara, Tom reste enfermé à côté de la pelle avec laquelle, dans le tableau final, il assomme Francis avant de lui ouvrir la bouche jusqu'à la déchirer, en utilisant les mêmes mots que Francis lui adressait lorsqu'il l'étouffait : «Tu me dis quand arrêter, mon homme » (58). Faute et fatum, vengeance rituelle et catharsis, rédemption : le drame familial de la mystification s'élève au rang des mythes tragiques et sur les vêtements ensanglantés de Tom se coagule la dimension sacrificielle de la pièce : sang de la naissance du veau, entrailles saignantes dans la fosse aux vaches mortes. Mais en même temps qu'il s'universalise, le discours sur l'homophobie, loin de se dépolitiser, se complexifie, puisque ce dénouement est également, pour reprendre le sous-titre des Feluettes, la répétition d'un drame homophobe ${ }^{12}$ : non seulement il reproduit à l'identique la violence hétérosexiste, mais il renoue aussi avec la nécessité de mentir, le mot de la fin, prononcé par Tom, étant un projet de mensonge : "Je dirai à Agathe que Francis est parti en ville rejoindre Sara " (58). Car il est écrit dans le placard qu'« il faut jamais dire la vérité » (57).

\section{À film à suspense, désirs en suspens}

7 Déconcertée par l'atmosphère qui règne à la ferme de l'imposture, la Sara de Bouchard ironise : « Dans le film, c'est-y moi la niaiseuse qui va se faire arracher la tête quand la musique va s'arrêter?» (Bouchard 2012: 49). Or, ce que Xavier Dolan se propose d'explorer dans son film, c'est moins le thème de l'homophobie en soi que l'amalgame de violence et désir qu'il implique. Tout en restant fidèle aux données fondamentales 
de l'histoire, il tient à en donner une version plus sobre et plus sombre - voire nocturne, quasi onirique : c'est endormi, la tête sur la table de la cuisine, qu'Agathe surprend Tom au début du film -, une version plus tendue et beaucoup moins verbeuse. À cette fin, il gomme tout effet comique de même que tout renvoi à la religion. Il atténue tout ce qui relève des conventions théâtrales, à commencer par le discours intérieur verbalisé de Tom: pas de voix off dans le film, compensée par l'introduction de plusieurs scènes intimes - en voiture ou autour du réfrigérateur, équivalent rétroéclairé du placard qu'il s'agirait de nettoyer-, des scènes aptes à véhiculer le désarroi du protagoniste et les fantasmes qui peuplent son silence. À sa première collaboration avec le directeur de la photographie André Turpin, le réalisateur met à profit toutes les ressources de son propre «maximalisme expressif» (Marshall 2016: 189) : montage syncopé, dont Dolan s'occupe lui-même, dominante picturale des prises de vues et alternance de plans larges et de close up. Il échappe au huis clos du théâtre filmé en multipliant les séquences d'extérieur et en privilégiant les espaces et les surfaces liminaires, portes, fenêtres et couloirs qui à la fois séparent et relient entre eux les « lieux-scènes » (Rivière Lechat $2016: 204)$. Il étire le temps - la pièce et le film ont plus ou moins la même durée, mais le temps raconté passe d'une semaine à un mois environ - et il segmente les douze tableaux de la pièce en une centaine de scènes filmées. Tantôt il élimine, tantôt il ajoute des éléments - pas de scène au cimetière, ni dans le coffre de la voiture ou en surplomb de la fosse aux vaches, mais nous assistons par exemple aux obsèques et à la visite chez le médecin - et il en disloque d'autres. Il cultive la prolepse, l'ellipse et l'implicite, les séquences sans dialogue du début étant éloquentes à ce propos. En donnant sans cesse à entrevoir le non-dit, Dolan alimente le mystère : il assouvit par là un désir de thriller dont participent le bruitage inquiétant et la bande originale composée par Gabriel Yared et suspendue entre Bernard Herrmann, le collaborateur de Hitchcock, et Mahler ${ }^{13}$, ainsi que les citations de Shining - le travelling initial - et de Psychose, sous la douche. Surtout, avec la collaboration de Bouchard, Dolan change la fin de l'histoire. Dans le film, Tom ne tue pas Francis; il l'abandonne à sa solitude de paria après avoir découvert - de façon autonome, en posant des questions au barman, et beaucoup plus tard que dans la pièce, où c'est Francis lui-même qui le lui raconte - le secret du garçon dont il entrevoit un moment le visage déchiré lorsqu'il s'arrête à une station-service. C'est par l'irruption de la violence dont l'Autre a été la victime que le protagoniste s'en sort, affirme le réalisateur ${ }^{14}$, quoiqu'on ait pu deviner dans les images conclusives du film - Tom empoignant le volant à un feu rouge de Montréal, son regard dans le rétroviseur - une hésitation, la tentation peut-être de faire retour, encore une fois, vers l'épicentre d'un désir aussi troublant qu'irrépressible (Marshall 2016: 206-207): feu vert aux interprétations.

8 Les modifications apportées dans le passage de la dominante verbale du théâtre à la dominante iconique du cinéma concernent également la construction, plus austère, des personnages. Par exemple, Agathe est privée dans le film des quelques détails qui, dans la pièce, lui sont fournis à propos des circonstances dans lesquelles son fils est décédé. La dernière apparition de la mère - autour de la boîte contenant des cahiers dont il n'a jamais été question dans le film, que Tom trouve aux pieds de son lit et qu'il emporte sans que le contenu en soit révélé - n'en devient que plus intense: son deuil miaveugle, mi-clairvoyant, son épuisement et son amertume ne détonnent qu'apparemment avec les figures maternelles hautes en couleurs des autres films de Dolan. Quant à Sara Thibaut - car dans le film les personnages ont un nom de famille, 
celui de Tom étant Kosovski-, elle n'est que la fausse fiancée du mort: on ne la rebaptise pas Ellen et on ne la fait pas parler anglais " comme une vache espagnole " (Bouchard 2012: 26). De même que l'homophobie de Francis résulte moins explicite, Tom est moins typisé, voire stéréotypé, en tant qu'homosexuel narcissique ou efféminé : nous voyons Francis le macho repasser ses propres chemises et c'est plutôt Tom qui l'insulte en le traitant de farmer attardé. De même que le contraste entre métropole et campagne s'estompe un peu, les identités, sans pour autant devenir interchangeables, sont dessinées de manière plus nuancée, plus fluide. Dans le film de Dolan, pas plus de cross-dressing ou de travestissement que d'alternance codique, mais beaucoup de code-mixing, d'enchevêtrement du français et de l'anglais : Taille-de-Fille, le nom à l'image de la «tantouze » donné par Francis au veau nouveau-né dans la pièce (Bouchard 2012 : 30,36), est changé, mais sur un ton moins moqueur qu'ému, en Culde-Bitch.

9 Si dans la pièce de Bouchard le bilinguisme relève de la fiction et de l'incommunicabilité, la plasticité rythmique et triviale du québécois joualisant, troué d'anglicismes, parlé dans le film ouvre le français à l'appropriation, à l'hybridation, à la compénétration avec l'anglais, en démystifiant par là tout monolinguisme, en brouillant les frontières et en déstabilisant le rapport entre dominant et dominé (Mandia 2014). Le transfert de la scène centripète à l'écran centrifuge ${ }^{15}$ entraîne d'ailleurs une dissémination des indices d'une québécitude que le réalisateur revendique, tandis qu'il conteste, lui aussi, la ghettoïsation qu'implique l'étiquette de cinéaste $\mathrm{LGBTQ}^{16}$. En témoignent les chansons - toutes canadiennes et, comme toujours chez Dolan, très éloquentes (Laverdière 2015) - qu'on écoute dans le film. La première, interprétée a cappella par la comédienne et chanteuse québécoise Kathleen Fortin, est Les Moulins de mon coeur, un classique français de 1968 par Michel Legrand et Eddy Marnay dont la traduction en anglais - The Windmills of Your Mind - a valu, la même année, l'Oscar de la meilleure chanson originale à The Thomas Crown Affair, un polar qui est aussi une histoire de séduction entre le malfaiteur et l'enquêtrice. Spéculairement, lors des funérailles de Guillaume et du karaoké en flash-back qui s'y entremêle, Mario Pelchat, originaire du Lac-Saint-Jean comme Bouchard, chante Pleurs dans la pluie, l'adaptation française, par le même Eddy Marnay, de Tears in the Rain de Desmond Child et Diane Warren, dont la version originale remonte à 1989. Le va-et-vient d'une langue à l'autre se poursuit jusqu'au générique, accompagné de Going to a Town du compositeur et interprète Rufus Wainwright : grandi à Montréal, homosexuel marié et coparent d'une fille, celui-ci se transforme en prima donna dès qu'il réincarne sur scène l'icône gay Judy Garland. "I'm so tired of you America » : dans cet hymne anti-Bush qui date de 2007, Wainwright chante le désenchantement et le déclin de l'empire américain, un peu comme le drapeau américain sur le blouson que Francis traîne dans la boue vers la fin du film devient le symbole de son aliénation socio-économique et culturelle. Dans le bois aux coyotes, au moment où Tom s'enfuit, la rage de Francis implose et ses menaces se muent en imploration. Mais Rufus Wainwright chante aussi, malgré l'intolérance réactionnaire dominante, les marges d'autonomie existentielle et affective de l'individu : «Ain't gonna be alone / I've got a life to lead, America ». Alors que, dans le bar où Francis a ouvert la bouche de Paul pour le faire taire, pendant que Tom l'enquêteur découvre le secret de la face déchirée, résonne un tube années 1980 de Corey Hart : «I wear my sunglasses at night "- «je porte mes lunettes de soleil la nuit »-, comme pour regarder sans être vu ce qui se cache dans l'obscurité du placard. Ainsi que le souligne Bill Marshall (2016) et que cette multiplication de suggestions 
croisées le confirme, Dolan aborde la question de l'identité québécoise dans la pluralité de ses composantes - nationale et régionale, linguistique et culturelle - et par une pluralisation déterritorialisante des références, voire une valorisation des interférences qui ne se constituent pas en un discours unitaire et totalisant, mais qui, bien au contraire, mettent en valeur l'hétérogénéité, les fluctuations, le métissage.

De même, l'orientation sexuelle ne détermine pas de relation figée et univoque à l'autre. Pour sensible qu'il soit au problème de l'homophobie - et la vidéo de 2013 pour la chanson College Boy du groupe Indochine l'atteste de façon extrêmement explicite -, dans Tom à la ferme Xavier Dolan semble déplacer l'accent de la culpabilité et de la duplicité intériorisées par l'homosexuel vers les ambivalences labyrinthiques du désir tout court; d'où la fin, moins tragique et définitive, du film. Encore une fois, et dès le début, la vérité inavouée s'affiche par écrit. Dans le prologue en plan rapproché, une main nerveuse griffonne au feutre bleu sur une serviette en papier le texte du monologue que, comme dans la pièce, Tom attribuera plus tard à la fiancée présumée du mort. Sa voix sans visage, encore sans sujet, marmonne quelques mots ici et là. Ainsi, aux phrases prévues par Bouchard s'ajoute celle-ci : «Maintenant ce qu'il nous reste à faire sans toi, c'est te remplacer. » Infaisable, le deuil comporte une forme de substitution. Mais qui est ce "nous »? C'est encore Tom et Guillaume, ou est-ce déjà Tom et Francis? Ce qui intéresse le plus Dolan dans ce drame de l'homophobie, c'est la dynamique psychologique, affective et sensuelle que déclenche la rencontre entre deux êtres en manque de repères, l'un et l'autre seuls, vulnérables et en quête d'expiation, de salvation. Et Dolan d'évoquer le syndrome de Stockholm, c'est-à-dire la stratégie d'adaptation et de survie qui consiste pour l'otage à s'identifier au ravisseur et à laquelle correspond, à rôles renversés, le syndrome dit de Lima, tous les deux largement exploités en littérature, en musique populaire et au cinéma ${ }^{17}$. Si Dolan réduit à un regard intense de Tom les attouchements dans le noir de la chambre qu'il partage avec Francis - «Tu me dis quand arrêter!» (Bouchard 2012: 43) -, dès le clin d'œil initial - dans une rue au fin fond de nulle part, la ferme des Longchamp se trouve au 69 , chiffre de la réversibilité sexy-, le réalisateur renforce la charge sexuelle de la soumission consentante à une domination jouissive, avec tout ce que celles-ci comportent de répulsion et d'attirance concomitantes et surtout de dépendance mutuelle ${ }^{18}$. C'est, bien sûr, la scène de bal, précédée dans le film par le défi de la prise de cocaïne, où Dolan fait d'une rumba un tango à son tour renversé en gotan sur la musique envoûtante du groupe international Gotan Project. Mais ce sont également la scène des toilettes de la salle communautaire, pendant la réception qui suit les obsèques ; la séquence, qui ne figure pas dans la pièce, où Tom, les cheveux longs de la couleur du maïs, se fait massacrer, puis cracher dans la bouche par Francis - « C'est ton choix d'être revenu ", "Tu vas marcher droit»- dans un champ de maïs qui devient l'emblème de ce thriller, car, au mois d'octobre, « un champ de maïs est comme un champ de couteaux »; et encore, l'étranglement, retardé et déplacé dans un extérieur nocturne par Dolan, qui en accentue le côté quasi orgasmique par le rétrécissement du format et le plan on ne peut plus rapproché. Alors que dans sa relecture cinématographique de Juste la fin du monde, Dolan rend évidente l'homosexualité de Louis, en contrevenant à la réticence de Lagarce, dans Tom à la ferme c'est donc en marge, voire en dehors, des normes, tant hétérosexistes qu'homosexuelles, que Francis et Tom interrogent les fluctuations de leur désir et que Dolan construit ses personnages. 


\section{Placards}

11 pas réduire à un parti pris cinématographique naturaliste ou à la seule logique du suspense, Dolan restitue donc une version encore plus ambivalente de l'histoire, qui va de pair avec une vision plurielle et mobile, performative et relativiste de l'identité tant linguistico-culturelle que sexuelle: en un mot, une version plus queer. À partir des mêmes préoccupations sur la persistance de la menace homophobe, Michel Marc Bouchard élabore un drame allégorique des tensions socio-culturelles que le métathéâtre complexifie et mythifie, alors que Xavier Dolan explore par le thriller les interstices de la relation à l'autre en déstabilisant la notion d'identité elle-même. De ce point de vue, la transposition de la scène à l'écran invite le spectateur à mesurer l'écart générationnel séparant le dramaturge et le réalisateur. Né en 1958 et auteur, dès 1984, d'une pièce sur l'homoparentalité, La Contre-nature de Chrysippe Tanguay, Michel Marc Bouchard a à peu près l'âge du père de Dolan, Manuel Tadros, auquel Xavier - 23 ans à l'époque du tournage - confie significativement le rôle du barman, le témoin de la violence de Francis qui, en dévoilant le mystère de l'isolement des Longchamp, passe le témoin à Tom. Sans exagérer la distance entre ces deux œuvres, due dans une très large mesure à la diversité des moyens expressifs employés, les versions dramatique et cinématographique de Tom à la ferme témoignent de deux sensibilités différentes, ou plutôt d'une sensibilité en mouvement sur la route qui a mené des descentes de police dans les bars gays au mariage pour tous, en passant par les années du deuil volé. La pièce de Bouchard relève d'une intention d'affirmation identitaire, problématisée et transfigurée à travers le motif de la mystification existentielle, alors que le film de Dolan adopte la consistance d'une identité fuyante, poreuse, à reformuler constamment. De la fiction identitaire, on glisse vers l'identité comme fiction.

Deux visions du placard en découlent, l'une plus sombre et suffocante, enfermée dans la répression du désir, l'autre moins étanche, plus élastique, où des fissures permettent d'entrevoir les contradictions du désir; deux visions moins concurrentielles que complémentaires sans doute, puisqu'elles tiennent toutes les deux à la fois du cauchemar et de l'épiphanie. Deux épistémologies néanmoins distinctes du closet, que Dolan prend au pied de la lettre dans la scène du Water Closet où il déplace l'imposition, par Francis, de Sara en tant que fiancée de Guillaume : il s'agit d'obliger Tom à parler, à feindre et à fabuler pour ne pas dire la vérité inavouable, dans une clandestinité que l'incursion d'un usager ne fait qu'érotiser davantage. "Je me souviens de ta voix", affirme Francis, "Je savais que tu débarquerais »: Tom est attendu ; il s'agit pour lui d'un retour à l'endroit, le placard, où il n'a peut-être jamais été, où il ne soupçonnait pas son amant menteur d'avoir été, alors que - ou, plutôt, justement parce que Guillaume, à l'âge de 16 ans, s'y était enfermé à jamais comme dans un tombeau, dans une crypte, de même qu'il avait enseveli ses cahiers dans une boîte. Or, le vivant remplace le mort, le mort revient dans le vivant, et les dénouements des deux intrigues s'avèrent révélateurs : celui de la pièce, tragique et libérateur, mettant au jour mais aussi reproduisant les mécanismes de la violence et du mensonge qui règlent le fonctionnement du placard en tant que circuit fermé ; celui du film, déchirant mais ouvert. Le métathéâtre de Bouchard dramatise le discours autour du placard ; la caméra de Dolan y pénètre, explore les échanges, les tensions désirantes qui le traversent et qui en font un espace d'élaboration et de transformation, un espace toxique, certes, mais

Itinéraires, 2019-2 et 3 | 2019 
dynamique. Lorsqu'Agathe sort enfin du placard la boîte contenant les cahiers de Guillaume et autres reliques de son fils comédien et martyr, le cinéaste place le spectateur à l'intérieur du placard, grâce à l'un de ces raccords symétriques alliant emboîtement des images et renversement de la perspective pour lesquels son style est tour à tour admiré et critiqué. C'est vers nous, dans le noir de la boîte, que les personnages regardent, de haut en bas : «C'est quoi ? ", demande Francis. Par la même prise de vue en contre-plongée, Dolan vide la boîte-placard dans laquelle il nous enferme et il nous oblige à fouiller dedans, à la remplir de nos propres impostures intériorisées, de nos propres désirs inavoués. En mode mineur, c'est peut-être encore une catharsis.

\section{BIBLIOGRAPHIE}

Bersani, Leo, 1998, Homos. Repenser l'identité, Paris, Odile Jacob.

Bovet, Jeanne, 2007, « Du plurilinguisme comme fiction identitaire », Études françaises, vol. 43, n 1, p. 43-62, [En ligne], https://id.erudit.org/iderudit/016297ar, consulté le 29 novembre 2019. DOI : 10.7202/016297ar

Brook, Peter, 2010, « Entrer simultanément dans les deux mondes », dans B. Picon-Vallin (dir.), Le film de théâtre, Paris, CNRS Éditions, p. 232-240.

Chabrol, Marguerite et Karsenti, Tiphaine (dir.), 2013, Théâtre et cinéma. Le croisement des imaginaires, Rennes, PUR.

Corriveau, Patrice et Daoust, Valérie (dir.), 2011, La Régulation sociale des minorités sexuelles. L'inquiétude de la différence, Québec, Presses de l'Université du Québec.

Defraye, Piet et MacDonald, Marylea, 1997, «Les Feluettes, un drame de la répétition », Dalhousie French Studies, $n^{\circ} 41$, p. 129-137.

Dyer, Kester, Lafontaine, Andrée et Massimi, Fulvia (dir.), 2016, Synoptique, vol. 4, $\mathrm{n}^{\circ} 2$, « Locating the Intimate within the Global: Xavier Dolan, Queer Nations and Québec Cinema », https:// synoptiqueblog.wordpress.com/4-2-locating-the-intimate-within-the-global-xavier-dolan-queernations-and-quebec-cinema/, consulté le 29 novembre 2019.

Eribon, Didier, 2012, Réflexions sur la question gay, nouvelle édition, Paris, Flammarion.

Eribon, Didier, 2015, Théories de la littérature. Système du genre et verdicts sexuels, Paris, PUF.

Godin, Jean Cléo et Lafon, Dominique, 1999, Dramaturgies québécoises des années quatre-vingt, Montréal, Leméac.

Helbo, André, 1997, L'Adaptation. Du théâtre au cinéma, Paris, Armand Colin.

Howe, Lawrence, 2006, " The Epistemology of Adaptation in John Greyson's Lilies ", Revue canadienne d'études cinématographiques/Canadian Journal of Film Studies, vol. 15, nº 2, p. 44-61.

Huffman, Shawn, 2007, « Entretien avec Michel Marc Bouchard », Voix et images, vol. 33, $\mathrm{n}^{\circ}$ 1, p. 15-25, [En ligne], https://id.erudit.org/iderudit/017524ar, consulté le 29 novembre 2019. DOI : 10.7202/017524ar 
Jullien, Danielle et Lévy, Joseph Josy (dir.), 2007, Homosexualités. Variétés régionales, Québec, Presses de l'Université du Québec.

Laverdière, Gabriel, 2015, «L'esthétique rock queer, de C.R.A.Z.Y à Xavier Dolan », Nouvelles vues. Revue sur les pratiques et les théories du cinéma au Québec, $\mathrm{n}^{\circ}$ 16, p. 1-18.

Lepastier, Joachim, 2014, « En fusion. Mommy de Xavier Dolan », Cahiers du cinéma, n 704, p. 6-9.

Mandia, Valérie, 2014, « Le septième art hors des frontières nationales : le pouvoir de la langue et de l'imaginaire culturel dans les films du cinéaste québécois Xavier Dolan », Francophonies d'Amérique, $\mathrm{n}^{\circ}$ 37, p. 105-132.

Marshall, Bill, 2016, « Spaces and Times of Québec in Two Films by Xavier Dolan », Nottingham French Studies, vol. 55, $\mathrm{n}^{\circ}$ 2, p. 189-208.

Rivière Lechat, Louise-Anne, 2016, « Tom à La Ferme, de la pièce au film : une réécriture audacieuse d'une tragédie en thriller », TrOPICS, n 3, p. 195-208.

\section{Corpus}

Bouchard, Michel Marc, Tom à la ferme. Le peintre des madones, Montreuil, Éditions Théâtrales, 2012.

Dolan, Xavier, Tom à la ferme, (c) 2013 Canada/France, Sons of Manual et MK2 en association avec ARTE/COFINOVA 9 avec la participation de CINÉ+ et du Centre national du cinéma et de l'image animée, (C) 2014 DVD MK2 TFI Vidéo.

\section{NOTES}

1. La pièce à été créée le 11 janvier 2011; mise en scène: Claude Poissant; scénographie : Romain Fabre ; distribution : Alexandre Landry (Tom), Lise Roy (Agathe), Éric Bruneau (Francis), Évelyne Brochu (Sara). Première édition: Montréal, Leméac, 2011. Prix de la dramaturgie francophone (SACD). Je remercie Laure Budzinski de la Compagnie Stasima qui, en 2016, a monté Tom à la ferme au Théâtre de la Manufacture de Nancy, ainsi que mon collègue Paolo Frassi.

2. Outre le site de l'auteur (http://www.michelmarcbouchard.com, consulté le 29 novembre 2019), voir Godin et Lafon (1999: 61-102), ainsi que le dossier contenant Huffman (2007).

3. Tourné à l'automne 2012, le film date de 2013 ; durée : 1 h 42. Le DVD MK2 TF1 (2014) contient un entretien avec Dolan où le réalisateur insiste sur la distance que son film a prise avec le drame de Bouchard. Si les choix narratifs et esthétiques qui distinguent l'adaptation cinématographique de la pièce de théâtre ne peuvent pas lui être attribués de façon univoque, Xavier Dolan peut néanmoins être considéré comme l'instance auctoriale principale du film, sachant qu'il s'agit là moins d'un parti pris théorique que d'une commodité opératoire.

4. Voir, par exemple, Eribon (2012 et 2015), ainsi que Bersani (1998).

5. En s'appuyant sur les travaux théoriques de Gérard Genette, de Jean-Marie Schaeffer et de Richard Saint-Gelais, Louise-Anne Rivière Lechat analyse la réécriture du tragique par Bouchard et l'appropriation des codes du thriller par Dolan en termes de 
«transmodalisation » et de «transfictionnalité »: ainsi, illustre-t-elle la «circulation intertextuelle" de motifs et de procédés alliant l'exploration de l'intime et sa dramatisation anxiogène (Rivière Lechat 2016 : 203, 205).

6. La coïncidence onomastique avec le nom du protagoniste rend la référence à saint Thomas particulièrement significative : «si je ne mets pas ma main dans la marque [...], je ne croirai pas... Touchez mes blessures » (30). Voir aussi : « AGATHE.- Amen, Francis, Amen » (57).

7. Voir l'entretien avec le dramaturge et le metteur en scène mené par Marie-Thérèse Fortin du Centre du Théâtre d'Aujourd'hui: https://www.youtube.com/watch? $\mathrm{v}=\mathrm{aqcV}$ wEbQdFM, consulté le 29 novembre 2019.

8. C'est cette phrase que Xavier Dolan regrette de ne pas avoir pu insérer dans les dialogues, car elle condense à ses yeux «toute l'émotion du film, toute sa fragilité, toute son actualité, tout son drame et toute sa prédisposition à la perversion » : section intitulée "De la scène à l'écran" du dossier de presse du film (http:// download.pro.arte.tv/uploads/DP-Tom-à-la-ferme.pdf, consulté le 29 novembre 2019). Le cinéaste s'y explique également au sujet de sa collaboration avec le dramaturge au cours de la scénarisation du film : chacun travaillant de son côté, Dolan a réélaboré à plusieurs reprises les versions envoyées par Bouchard. Le rythme de ce va-et-vient s'est intensifié au cours de l'été 2012.

9. Voir Defraye et MacDonald (1997) et, sur l'adaptation cinématographique de 1996, Howe (2006).

10. Le dramaturge mime l'obsession de Tom dans le « Mot de l'auteur » : "Chaque jour, des victimes injuriées, ostracisées, violentées, moquées, humiliées, blessées, battues, taxées, souillées, isolées, bafouées » (9).

11. Ces questions sont abordées sur les modes du conflit, du contact et de la rencontre intime au sujet d'autres pièces québécoises contemporaines, dont The Dragonfly of Chicoutimi de Larry Tremblay, dans Bovet (2007).

12. L'auteur affirme avoir tenté "plusieurs fins heureuses, mais les œuvres réconciliatrices dans leur résolution sont faites de morale à consommer sur place et nous déresponsabilisent face aux solutions des conflits » (9).

13. Dans la section «Travailler avec Gabriel Yared» du dossier de presse du film, Dolan parle de musique «romantico-panique»: http://download.pro.arte.tv/uploads/DPTom-à-la-ferme.pdf (consulté le 29 novembre 2019).

14. Parmi les nombreux entretiens qu'on peut visionner en ligne, voir la conférence de presse à l'occasion de la Mostra del Cinema de Venise 2013, où la critique internationale a décerné à Tom à la ferme le prix FIPRESCI, https://www.youtube.com/watch?v=7Xaou6MPw0 (consulté le 29 novembre 2019).

15. «La suggestion se fait au cinéma par la rupture, par le passage, par l'alternance des images, et non pas à l'intérieur de l'image même comme au théâtre » (Brook 2010 : 239; voir aussi Helbo 1997 et Chabrol et Karsenti 2013).

16. Notamment à l'occasion de la Queer Palm attribuée, au Festival de Cannes 2012, à Laurence Anyways, histoire d'une transition d'homme à femme et de l'amour qui ne cesse d'unir cette transgenre et la femme de sa vie. Voir Dyer, Lafontaine et Massimi (2016).

17. C'est le cas, en 1975, deux ans après la prise d'otages dans la capitale suédoise d'où le syndrome tire son nom, du film, riche en résonances politiques, de Sidney Lumet Un 
après-midi de chien (Dog Day Afternoon), où le projet de dévaliser une banque de Brooklyn a pour but de financer une opération de changement de sexe.

18. À ce propos, il est significatif de souligner que, lors du tournage, l'acteur interprétant le rôle de Francis n'avait ni vu ni lu la pièce de Bouchard et que Dolan ne lui a pas conseillé de le faire. Voir l'interview avec Pierre-Yves Cardinal: https:// www.youtube.com/watch?v=X4rmroYmWgQ\&t=11s (consulté le 29 novembre 2019).

\section{RÉSUMÉS}

Écrite en 2010 et créée à Montréal en 2011, la pièce en douze tableaux Tom à la ferme du dramaturge québécois Michel Marc Bouchard a été portée à l'écran en 2013. L'auteur lui-même a travaillé au scénario en collaboration avec le réalisateur, coproducteur et interprète du film Xavier Dolan. C'est ainsi que ce drame de l'homophobie et du mensonge intériorisé, riche en dispositifs métathéâtraux et alternant la violence et le comique, devient un thriller psychologique de la domination érotisée. Comportant plusieurs déplacements d'accents narratifs et esthétiques, cette adaptation donne lieu, dans cet article, à une comparaison concernant la manière dont la pièce, puis le film, posent les questions de l'identité culturelle, linguistique et homosexuelle. Dans le passage de la scène à l'écran, la mystification existentielle cède le pas aux fluctuations de la répulsion et de la fascination indémêlables : de l'affirmation d'une identité problématique on glisse vers la problématisation de toute affirmation identitaire.

Written in 2010 and first staged in Montreal in 2011, Michel Marc Bouchard's Tom à la ferme is a highly metatheatrical tragicomedy of homophobia and existential mystification. In 2013, Xavier Dolan's cinematographic adaptation transforms it into a psychological thriller which explores the fascination with violence and blurs the boundaries between domination and dependence. The playwright and the director having collaborated on the screenplay, the present comparison aims at showing how Bouchard's play, then Dolan's film, address the issues of cultural, linguistic and homosexual identity, thereby questioning both the fiction of identity and identity as fiction. This stage-to-screen adaptation involves some slight or substantial modifications as well as a pluralisation of references which, by emphatising the fluctuations and contradictions of desire, problematize the very notion of stable identity.

\section{INDEX}

Mots-clés : cinéma, homosexualité, homophobie, théâtre, métathéâtre, Québec

Keywords : cinema, homosexuality, homophobia, theatre, metatheatre, Quebec

\section{AUTEUR \\ STEFANO GENETTI}

Université de Vérone 